\title{
JUAN SMITH SINNOT Y ANTONI DE MARTÍ I FRANQUÈS EN LA VACUNACIÓN DE TARRAGONA DEL AÑO 1801
}

\author{
Agustí Camós \\ Centre d'Història de la Ciència - UAB. \\ agusti.camos@gmail.com \\ ORCID iD: https://orcid.org/0000-0002-9054-660X
}

Recibido: 21 enero 2017; Aceptado: 26 junio 2017.

Cómo citar este artículo/Citation: Camós, Agustí (2018), "Juan Smith Sinnot y Antoni de Martí i Franquès en la vacunación de Tarragona del año 1801", Asclepio, 70 (1): p209. https://doi.org/10.3989/asclepio.2018.02

RESUMEN: En la primavera del año 1801 se produjo en Tarragona una de las primeras campañas de vacunación antivariólica de la península ibérica. El destacado científico tarraconense Antoni de Martí i Franquès conocido especialmente por sus trabajos químicos y botánicos, también se interesó por problemas médicos y participó como experto en la citada vacunación probablemente influido por Francesc Salvà y Francesc Piguillem, facultativos amigos suyos favorables a esta nueva técnica preventiva. El responsable de la vacunación fue el ingeniero de la Armada y director de las obras del puerto de Tarragona Juan Smith Sinnot, sobre el que se aportan algunos datos biográficos que permiten comprender su interés por la vacunación contra la viruela. Smith en su obra Progresos de la vacina en Tarragona (Tarragona, 1801) analiza distintos aspectos de esta inoculación y explica algunos de sus intentos por difundir esta nueva técnica. En el artículo también se estudia la relación de Martí con Smith y los datos que éste último aporta sobre el viaje de Martí por las principales ciudades europeas realizado entre 1800 y 1801.

PALABRAS CLAVE: Tarragona; España; Vacuna; Juan Smith Sinnot; Antoni de Martí i Franquès.

\section{JUAN SMITH SINNOT AND ANTONI DE MARTÍ I FRANQUÈS IN THE VACCINATION OF TARRAGONA OF THE YEAR 1801}

ABSTRACT: One of the first smallpox vaccination campaign in the Iberian Peninsula was carried out in Tarragona, during the spring of 1801. The outstanding scientist Mr Antoni de Martí i Franquès from Tarragona, actively participated in this vaccination as an expert. Besides his chemical and botanical work he also was very interested in medical problems, probably influenced by his friends, the Doctors Francesc Salvà and Francesc Piguillem who were favourable to this preventive and then, new technique. The responsible for the vaccination was a Navy engineer and director of the Tarragona Port works, Mr Juan Smith Sinnot. Here is provided some biographical data supporting his interest on the vaccination against smallpox. From this experience, Mr Smith published the "Progresos de la vacina en Tarragona" (Tarragona, 1801) in which different technical aspects, such as the inoculation, were analysed. Here I analyse the Smith's interest to spread this new technique and the relationship between Martí and Smith providing some information about the Marti's traveling through the main European cities during 1800 and 1801.

KEY WORDS: Tarragona; Spain; Vaccine; Juan Smith Sinnot; Antoni de Martí i Franquès. 


\section{INTRODUCCIÓN}

El descubrimiento de la vacuna antivariólica realizado por Edward Jenner a finales del siglo XVIII, constituye uno de los hechos más destacados en la lucha contra las enfermedades infecciosas. Por ello, desde hace años numerosos historiadores de la ciencia han investigado tanto el propio proceso del descubrimiento como el de su rápida llegada a los distintos territorios y países. En este sentido en los últimos decenios diversos autores han investigado sobre la llegada y la apropiación de esta nueva técnica en el Estado español, entre los que destacan Manuel Riera y José Rigau-Pérez (1992), Guillermo Olagüe y Mikel Astrain (1994, 1995, 2004), José Luis Duro (2014) y José Tuells (2015).

Gracias a sus investigaciones conocemos que la primera vacunación en el Estado se produjo en Puigcerdà en diciembre del año 1800, siendo el responsable de la misma el doctor Francesc Piguillem quién se convirtió en uno de los grandes divulgadores de la nueva técnica. Gracias al material suministrado por Piguillem pocos meses más tarde se producía en Tarragona una masiva vacunación poco conocida, que presenta algunas características bastante singulares entre las que destaca que proceso fuese liderado por un brigadier de la armada de raíces irlandesas llamado Juan Smith Sinnot. Se trata de un interesantísimo personaje polifacético sobre el que solo se han realizado unos pocos estudios, como los de Manuel Riera (1992) y Josep Adserà (1993). Otro personaje que tampoco era médico y que tuvo un destacado papel en la vacunación en Tarragona es algo más conocido, fue el notable naturalista y químico tarraconense Antoni de Martí i Franquès.

Para escribir el artículo se ha recabado el mayor número de datos que permitan comprender las razones que llevaron brigadier Juan Smith, cuando era director de las obras del puerto de Tarragona, a realizar un enorme esfuerzo para conseguir que se vacunaran más de un millar de habitantes de Tarragona y poblaciones circundantes. También se han investigado las razones que pudieron llevar Martí Franquès a interesarse por esta nueva técnica médica hasta el punto de convertirse en un experto según Smith, para lo que se han utilizado diversos estudios sobre este interesante personaje. También se ha analizado la forma en que se produjo esta vacunación en la primavera del año 1801, así como los esfuerzos realizados por Smith para dar a conocer esta técnica en otros territorios peninsulares. Para realizar esta investigación hemos dispuesto de una obra fundamental escrita por Smith el mismo año de la vacunación, Progresos de la vacina en Tarragona, de otras fuentes primarias como distintos documentos de la Real Sociedad Económica de Amigos del País de Valencia, así como de diferentes obras sobre la vacunación publicadas por otros autores españoles en los primeros años del siglo XIX. También han sido fundamentales los trabajos sobre las primeras vacunaciones realizadas en el Estado publicados en los últimos decenios por historiadores citados anteriormente.

El artículo se inicia con el estudio de las muy poco conocidas relaciones de Martí Franquès con la medicina. A continuación se exponen todos los datos relevantes que se han localizado sobre la vida y el trabajo de Juan Smith. Después se analiza la posición de distintos médicos y cirujanos amigos de Martí Franquès sobre la nueva técnica de la vacunación, puesto que pudieron influir en el interés mostrado por este químico y naturalista. Luego se estudia cómo se produjo el proceso de vacunación en Tarragona y poblaciones próximas, el papel desarrollado por Martí Franquès, y se analiza hasta qué punto tuvieron éxito los intentos de Smith por divulgar la técnica de la vacuna a otros puntos del territorio español. Finalmente se señalan las principales conclusiones.

\section{MARTÍ Y LA MEDICINA EN BARCELONA}

Antoni de Martí i Franquès (Figura 1) nacido en el año 1750 en Altafulla, una población costera tarraconense, y muerto en Tarragona el año 1832, ha sido uno de los más grandes científicos catalanes. Sin embargo nos legó muy pocos datos sobre su pensamiento y sus investigaciones debido a su personalidad y las circunstancias que rodearon su vida, en particular por su miedo a la reacción de las autoridades eclesiásticas (Camós, 2013b). Como destacado científico ilustrado dedicó su actividad a cultivar diversos campos de la ciencia, como la química, la botánica, la geología, la meteorología o arqueología, destacando especialmente por sus investigaciones sobre la composición del aire, y por las dedicadas a la reproducción de los vegetales y a la generación espontánea. A pesar de que solo presentó cinco memorias en las academias a las que pertenecía y que únicamente publicó un trabajo en vida, su influencia en el mundo científico catalán y español fue mayor de lo que a menudo se ha supuesto (Camós, 2016a).

En Barcelona a finales del siglo XVIII y principio del XIX había varías instituciones donde se debatían las novedades científicas que llegaban desde Europa en las que participaban muy activamente médicos y cirujanos. Entre ellas destacan tres, la Real Academia 
Médico Práctica, la Real Academia de Ciencias y Artes, y el Real Colegio de Cirugía; Martí fue miembro de las dos primeras. Es bastante lógico que perteneciera a la academia de ciencias dado el gran trabajo que sabemos que desarrolló en diversas áreas científicas. Es más sorprendente su pertenencia a una academia médica aunque lo fuese como socio libre, una categoría reservada a aquellos que no siendo médicos hubiesen destacado en alguna de las ciencias que tenían relación con la medicina. En el caso de Martí los trabajos sobre la composición del aire y los dedicados a la botánica y a la generación de los organismos, se podían relacionar con el estudio de las causas de las enfermedades, de su transmisión y de su tratamiento.

Martí fue nombrado socio libre de la academia de medicina en 1790, y podemos hacernos una idea del prestigio que ya tenía en el mundo médico barcelonés si consideramos algunas de las personalidades que también fueron nombradas socios libres durante el mismo año. Entre ellos encontramos al botánico y presidente de la Real Sociedad de Medicina de París Mathieu Tillet, al catedrático del Jardín Botánico de Madrid Antoni Palau Verdera, al prestigioso químico Joseph Louis Proust y al farmacéutico y químico Pedro Gutiérrez Bueno (Quintana, 1935, p. 155). Fue en esta academia donde presentó el único trabajo que imprimió en vida, la memoria "Experimentos y Observaciones sobre los sexos y fecundación de las plantas", donde refutaba a través de un laborioso trabajo experimental la tesis del científico y sacerdote italiano Lazzaro Spallanzani, según la cual algunas plantas podrían producir semillas a partir únicamente de la parte femenina de la flor.

Martí disfrutó de la amistad de varios miembros de la Academia Médico Práctica que fueron importantes defensores de la vacunación, entre los que destacan Francesc Salvà y Francesc Piguillem. Ignacio María Ruiz de Luzuriaga, un personaje central en la introducción de la vacunación en España, así lo reconocía en una carta que escribió en 1801 recogiendo los primeros pasos de la vacuna en el Estado (Olagüe y Astrain, 1994, p. 318), y que iba dirigida a otro de los socios libres de esta academia, Proust (Tuells, 2015).

Salvà era uno de los médicos más prestigiosos de Barcelona y amigo íntimo de Martí (Quintana, 1935, p. 84). En dos de las tres sesiones de la academia médica que se dedicaron a la memoria de Martí sobre la reproducción de las plantas, fue Salvà quién la leyó en ausencia de su autor, y también fue él quien realizó muchas de las gestiones necesarias para su publicación. Precisamente en una carta de Martí re-
Figura 1. Retrato al óleo de Antoni de Martí i Franquès pintado por el académico Josep Arrau, que se conserva en la Reial Acadèmia de Ciències i Arts de Barcelona (circa 1835).

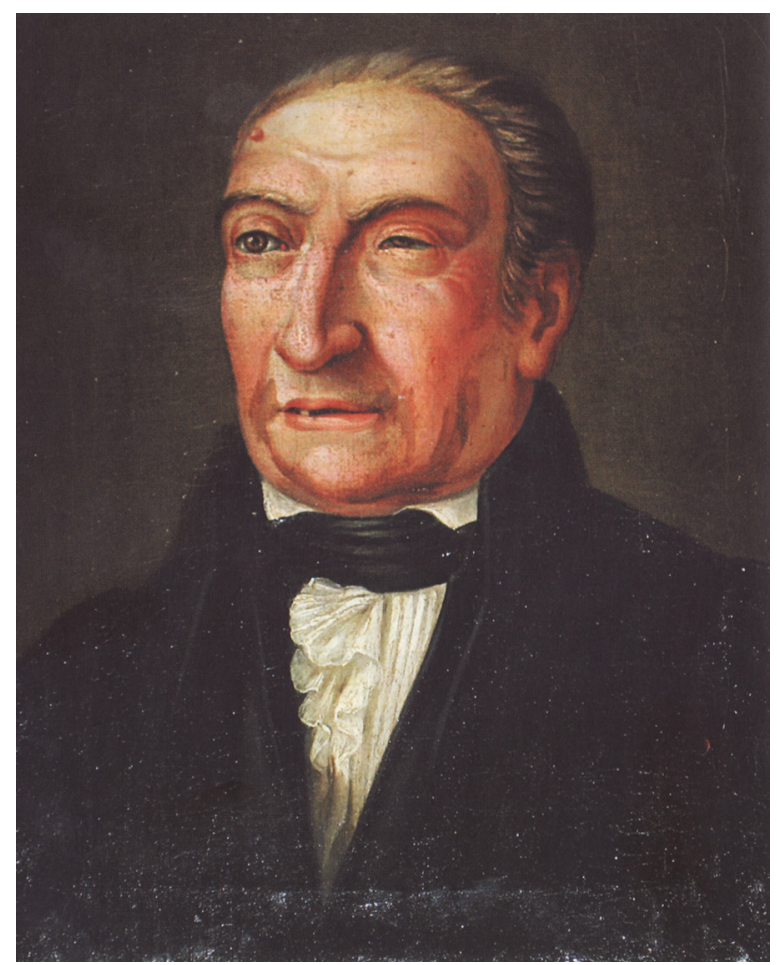

lacionada con las gestiones para la publicación de la memoria encontramos una clara manifestación de amistad y confianza cuando refiriéndose a algunos cambios hechos por Salvà escribe: "ya le tengo dicho que $\mathrm{Vm}$. tiene de mi parte todas las facultades para hacerlo sin darme razón de los motivos» (Quintana, 1935, p. 158). Se ayudaron mutuamente en algunos trabajos experimentales, como en los de Salvà sobre el galvanismo (Salvà, 1876, p .47), o en los de Martí sobre la composición del aire (Martí, 2011, p. 67) y sobre la generación espontánea (Quintana, 1935, p. 186). También conocemos que Salvà le pidió a Martí que revisara su trabajo sobre las aguas sulfúreas artificiales antes de imprimirla (Janer, 1832, p. 51-52). Por todo ello debemos concluir que además de una íntima amistad también compartían una gran confianza en sus respectivas capacidades experimentales y científicas. Dos de las preocupaciones de Salvà como médico fueron el estudio de los procesos epidémicos atribuidos a la fiebre amarilla y la defensa de los métodos preventivos de la viruela; sabemos que Martí investigó sobre esta enfermedad y comprobaremos que colaboró en la primera vacunación en Tarragona. 
Otro destacado médico amigo de Martí fue Francesc Piguillem, quién realizó las primeras vacunaciones en el Estado español en Puigcerdà, la población donde había nacido. Era uno de los colaboradores botánicos de Martí (Quintana, 1985, p. 73), y junto su hermano Joseph que era también médico, formaba parte de una de las vías de entrada de los libros que Martí recibía del extranjero (Quintana, 1935, p. 249). Asimismo Martí tenía en su biblioteca la obra de Foucroy traducida por Piguillem, Filosofía Química, o verdades fundamentales de la Química Moderna, dispuestas con nuevo orden (Quintana, 1935, p. 277).

Francesc Carbonell, académico e igualmente amigo de Martí, también participó en el debate sobre la vacuna. Aunque recordado especialmente por sus trabajos farmacéuticos y químicos, también ejerció la medicina y tuvo una considerable actividad en la academia médica barcelonesa. En la traducción de la obra de Jean-Antoine Chaptal, La Chimie appliquée aux arts, después de elogiar la labor científica de Martí se refirió a "la amistad con que me honra este sabio» (Chaptal, 1816 III, p. 140-141). Con motivo de la muerte de Martí, Carbonell dirigió una sentida carta de condolencia a su hijo (Quintana, 1935, p. 271), y fue también él quien leyó en la academia la necrología de Martí inspirada en lo que había escrito previamente el clérigo Fèlix Torres Amat (Torres Amat, 1836, p. XXII). Dicha necrología se publicó posteriormente en el Diario de Barcelona con la firma de Carbonell.

Otros dos amigos de Martí pertenecientes a la comunidad sanitaria barcelonesa que también participaron en los primeros debates en torno a la aceptación de la vacunación, fueron los cirujanos Josep Torner y Antoni Bas. Ambos pertenecían al Real Colegio de Cirugía. Torner fue profesor titular del colegio de cirugía, cirujano mayor del Hospital de la Santa Creu, y uno de los colaboradores botánicos de Martí (Quintana, 1985, p. 72). Bas fue profesor de botánica del colegio de cirugía y responsable del jardín botánico mientras dependió del colegio, institución desde la que le hizo llegar a Martí numerosas especies vegetales (Quintana, 1985, pp. 65-71).

Algunas de las ideas de Martí relacionadas con la medicina aparecen en las últimas páginas de su famosa memoria "Sobre la cantidad de aire vital que se halla en el aire atmosférico y sobre los varios métodos de conocerla", donde hizo uno de los cálculos más precisos de su tiempo sobre la composición del aire que tuvo una notable repercusión en Europa (Grau, 2011, pp. 34-40). Martí al demostrar experimentalmente la constancia en las proporciones de oxígeno y nitrógeno del aire en las más diversas condiciones, afirma con rotundidad que por tanto estas supuestas variaciones no podían ser la causa de las enfermedades tal como proponían muchos médicos de su tiempo (Martí, 2011, p. 67). En cambio, coincide con la mayoría de sus contemporáneos en relacionar el origen de las enfermedades con los aires provenientes de zonas pantanosas de donde se desprendería una "substancia incógnita" capaz de alterar la salud (Martí, 2011, p. 68). También conocemos que investigó sobre la fiebre amarilla en los primeros años del siglo XIX, en el marco del gran debate que se desarrolló en Barcelona a raíz de brote que se produjo en el puerto de la ciudad en otoño del año 1803 (Camós, 2016b).

Otra fuente que proporciona algunas ideas del pensamiento de Martí sobre las enfermedades epidémicas la encontramos en la obra de Llorenç Presas Guerra a muerte al cólera morbo asiático y al Oidium Tuckery, publicada dos décadas después de su muerte. Presas conoció el pensamiento científico de Martí a través de su maestro Agustí Yañez y del estudio de sus manuscritos, y relacionó el origen de las "sustancias incógnitas" causantes de las enfermedades con la generación espontánea refiriéndose reiteradamente a las ideas del científico de Altafulla, quién defendía la existencia de este proceso y creía que era capaz de controlar sus resultados experimentalmente (Camós, 2013a).

\section{JUAN SMITH SINNOT}

La vacunación en la ciudad de Tarragona fue liderada por un personaje con una formación ciertamente alejada de la medicina, el Brigadier, Capitán de Navío e Ingeniero Jefe de la Real Armada Juan Smith Sinnot. Había nacido en Madrid en el año 1757 siendo sus padres irlandeses, Miguel Smith natural de Dummore y Antonia Sinnot nacida en Werbof (Delgado, 1985, p. 62). A los diez años quedó huérfano puesto que su padre murió en 1766 y su madre al año siguiente. A los 18 años residiendo en El Ferrol sufrió las viruelas sin poder contar con el consuelo de su familia, lo que debió marcarle profundamente (Riera y Rigau, 1992, pp. 294-295). En estos años también vivía en El Ferrol el médico militar irlandés Timotheo O'Scanlan, uno de los más firmes partidarios de la variolización, es decir la inoculación cutánea de líquido de la pústula de un enfermo de viruela humana para prevenir la enfermedad, que entre 1784 y 1792 publicó tres trabajos de difusión de esta técnica (Amenedo, 2010, p. 10). Siendo ambos personajes ilustrados y con raíces irlandesas, es muy probable 
que se conocieran e incluso que O'Scanlan le atendiera cuando sufrió las viruelas puesto que ocupó el puesto de primer médico del Hospital Real de Ferrol desde 1766 hasta finales de 1777. O'Scanlan realizó numerosas variolizaciones en la ciudad desde el año 1771 que causaron un gran impacto, por lo que Smith hubo de conocer de primera mano este método de prevención de la grave enfermedad que el mismo había sufrido (Amenedo, 2010, pp. 14-19).

Después de residir en El Ferrol, en 1791 lo encontramos en Cartagena (Delgado, 1985, p. 62), en 1793 como brigadier ingeniero jefe en Cádiz, y más tarde comisionado en Valencia (Pereyra, 1800, pp. 9 y 53). En el año 1799 fue nombrado director de las obras de ampliación del puerto de Tarragona (Figura 2). Era un prestigioso ingeniero de puertos que ya se había encargado de las obras del puerto de El Grau en Valencia, y que también elaboraría y dirigiría el proyecto de ampliación del puerto de Barcelona (Adserà, 1993, p. 33). En el año 1807 fue ascendido a jefe de escuadra de la armada por lo que fue trasladado a Cartagena, pero volvió a Tarragona al año siguiente al ser nombrado gobernador interino $y$ posteriormente corregidor, residiendo de nuevo en la ciudad hasta su muerte el 17 de marzo de 1809 (Escoda, 2008, pp. 69-72).

Durante los casi diez años que estuvo en la ciudad de Tarragona desplegó una notable actividad. Además de dirigir las obras de ampliación del puerto desarrollando un nuevo proyecto más ambicioso, fue el diseñador de una nueva población en la marina tarraconense y de las fortificaciones que la protegían, solucionó el problema del abastecimiento de agua al puerto, y participó intensamente en la organización de la visita de Carlos IV. Según el historiador Josep Adserà era un hombre inteligente, con gran experiencia y capacidad de trabajo, muy humano en el trato con los trabajadores y presidiarios, gestionando por ejemplo las pensiones de viudedad y de invalidez que se producían a consecuencia de los accidentes en las obras del puerto (Adserà, 1993, p. 11). Esta sensibilidad también se manifestó en su preocupación por los problemas sanitarios, concertando la asistencia médica de los accidentados en las obras con el Hospital de Sant Pau i Santa Tecla, estableciendo mejoras en la higiene y la alimentación de los trabajadores presidiarios (Riera, 1992, pp. 284287), o recomendando al Ayuntamiento la construcción urgente de un cementerio fuera de la población a raíz de la epidemia que se produjo en Tarragona en el año 1809 (Adserà, 1993, p. 58).
Figura 2. Plano de los proyectos de una nueva población de la marina de Tarragona y de la ampliación del puerto realizados por Juan Smith Sinnot, que se encuentra en la Biblioteca Pública Arús de Barcelona.

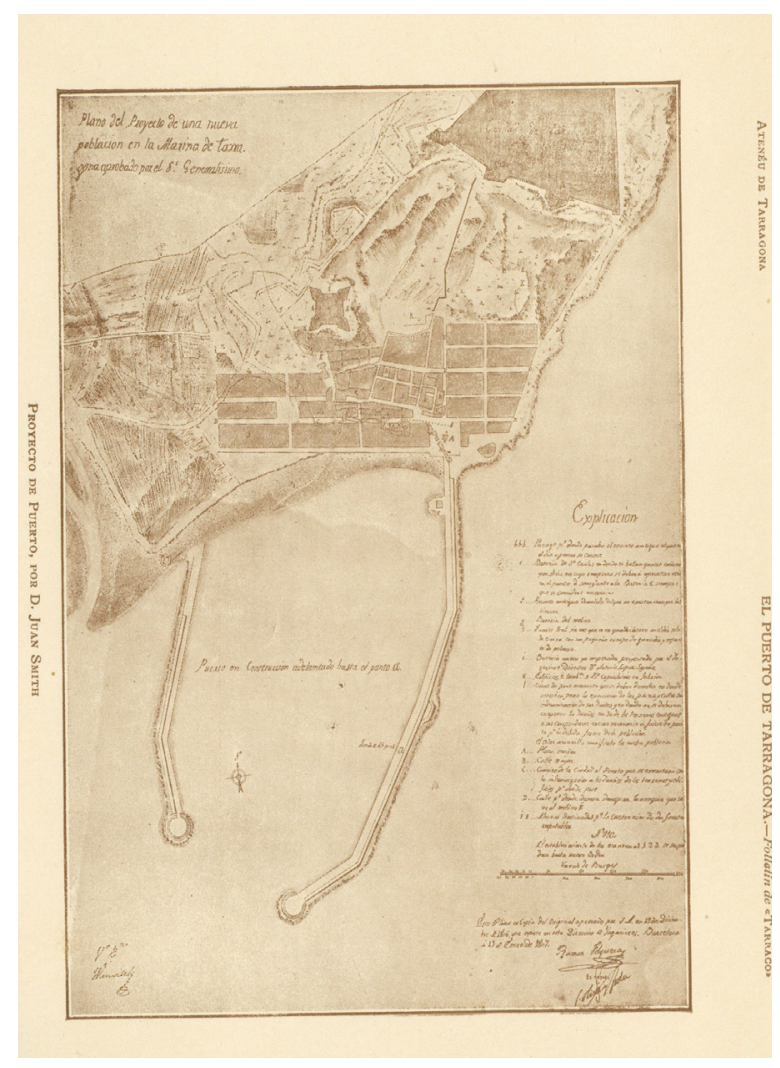

Lo que es indudable es que Juan Smith dejó perdurable recuerdo en la ciudad de Tarragona. Prueba de ello es que en el año 1860, medio siglo después de su muerte, se le escogió como uno de los personajes que tenían que figurar en la fachada del Ayuntamiento, aunque no había nacido en la ciudad y había vivido en ella tan solo un decenio (Hernández y Morera, 1865). Hay que añadir que en el año 1993, casi dos siglos después de su muerte, se trasladaron los restos de Smith a un mausoleo construido expresamente en el cementerio que él había promovido, haciendo realidad un acuerdo del pleno de Ayuntamiento republicano de diciembre de 1931 (Adserà, 1993, p. 12).

Además de ser un calificado ingeniero se interesó por otros muchos campos del conocimiento. Tuvo una notable actividad en arqueología, siendo académico correspondiente de la Real Academia de la Historia de Madrid. A dicha institución envió dos idolitos hallados en las excavaciones del puerto de Tarragona al demoler el convento de Capuchinos 
(Maier, 2003, p. 112). Entabló amistad con el arqueólogo francés Jean Baptiste Le Chevalier durante su estancia en Tarragona en los años 1804 y 1805, tal como puede desprenderse a través de diversas cartas del epistolario de Martí (Quintana, 1935, pp. 263-267). Además parece que ayudó a Martí a elaborar unos precisos y detallados planos de las murallas de la ciudad de Tarragona, que el naturalista de Altafulla envió al especialista en monumentos ciclópeos Louis-Charles-François Petit-Randel, amigo de Le Chevalier (Quintana, 1985, p. 78).

Otra muestra de sus amplios intereses fue la traducción de dos destacadas obras. La primera, Disertación Química sobre la transpiración y la respiración de Lavoisier, publicada en Valencia en 1797, pone de nuevo de manifiesto su interés por temas relacionados con la medicina. Se trata de dos memorias que Lavoisier escribió con su colaborador Armand Seguin en los años 1789 y 1790, que llevaban por título Premier mémoire sur la respiration des animaux y Premier mémoire sur la transpiration des animaux. La segunda obra, Principios de economía política, se publicó en Madrid en el año 1800 siendo la traducción de la obra del fisiócrata suizo Jean Herrenschwand, De l'economie politique moderne. Discours fondamental sur la population, publicada en Londres en el año 1786 y reeditada en París en 1795.

Estas traducciones también nos acercan a comprender su posicionamiento ideológico al analizar sus dedicatorias. La obra de Lavoisier la dedicó al prestigioso matemático Gabriel Ciscar, también miembro de la armada y destacado liberal, que después de participar en la guerra de la Independencia junto a las tropas españolas, y sufrir diversas vicisitudes en los años siguientes, acabó exiliado en Gibraltar debido a sus convicciones liberales. La amistad que le unía a Ciscar explica que fuera Smith quien el 21 de noviembre de 1797 entregara a la sociedad de amigos del país de Valencia tres obras remitidas por el matemático ${ }^{1}$. El libro de Herrenschwand lo dedicó al político ilustrado Mariano Luis de Urquijo, con quien mantuvo una muy buena relación mientras fue secretario de estado (Adserà, 1993, p. 26). Urquijo fue un destacado afrancesado que participó en el gobierno de José Bonaparte, y que se refugió en Francia tras la Guerra de la Independencia. El posicionamiento ideológico de Smith debió situarse entre el de un convencido liberal fiel a la monarquía española como Ciscar, y el de un afrancesado como Urquijo.

Esto explicaría su extraño comportamiento en los primeros años de la guerra de la Independen- cia. Cuando el 25 de mayo de 1808 el ayuntamiento de Tarragona lo nombró como representante en las Cortes que tenían que celebrarse en Bayona, Smith después de muchas dudas aceptó para poco después rehusar definitivamente el nombramiento, probablemente para alejarse de la política dictada por los franceses. No obstante, unos días más tarde aceptó el cargo de corregidor. Poco después permitió la entrada de las tropas francesas en la ciudad al mando del general Joseph Chabran, que de hecho fue quien le llevó su nombramiento como corregidor, haciendo caso omiso de las llamadas a la resistencia realizadas por un sector de la población tarraconense. Más tarde no envió refuerzos a las tropas españolas comandadas por el general Théodor Reding en la batalla que se libró contra las tropas francesas en las cercanías de Valls. A partir de aquel momento Smith no acudió a ninguna Junta Gubernativa ni presidió más el Ayuntamiento (Adserà, 1993, p.63), y Reding nombró en su lugar a Carlos Mesina como corregidor interino de Tarragona un par de meses antes de su muerte (Rovira, 2011, pp. 13-18).

Durante estos años de residencia en Tarragona mantuvo una notable actividad en los círculos ilustrados y de poder, estableciendo por tanto amistad con Martí, uno de sus prohombres. Martí estuvo muy vinculado a la ciudad aunque mantuvo su residencia en Altafulla hasta 1798. En este año se trasladó definitivamente a Tarragona instalando allí su gabinete y su magnífica biblioteca, por lo que en los años en que Smith vivió en Tarragona Martí también tenía allí su residencia habitual. Ambos participaron en las gestiones para que se hiciera una carretera entre Tarragona y Lleida (Quintana, 1935, p. 260), así como en un proyecto técnico-científico para la construcción de una máquina para subir agua (Quintana, 1992, p. 85). También colaboraron en uno de los mayores proyectos científicos de su época, la medición de la longitud del meridiano terrestre. Por otra parte Smith aparece en diversas cartas del epistolario de Martí, y en su biblioteca figura un ex-libris del brigadier (Quintana, 1935, p. 273).

Un punto importante de conexión entre Martí y Smith fue el gran interés que mostró la familia de Martí por las obras de ampliación del puerto de Tarragona, debido probablemente a la gran importancia que tenía para su actividad comercial. Así lo demuestra el substancial donativo que hizo el padre de Martí en 1790 para financiar las obras, que el propio Antoni Martí comunicó al Ayuntamiento. Algunos autores relacionan este donativo con la concesión 
del título de nobleza a la familia, que a partir de entonces introdujo la partícula "de" en su apellido (Sánchez Real, 1998, pp. 53-59).

También tenían intereses comunes en química y economía. La traducción de Smith de la obra de Lavoisier aborda uno de los temas sobre los que más investigó Martí, la química del aire. Smith se interesó por la economía como lo demuestra la traducción de la obra de Herrenschwand, mientras que Martí también se interesó por la economía política, como lo atestigua que en su biblioteca tuviera la traducción de $A n$ Inquiry into the Nature and Causes of the Wealth of Nations de Adam Smith realizada por Joseph Alonso Ortiz (Quintana, 1935: 283).

Asimismo como ilustrados, ambos también mostraron un gran interés por la enseñanza participando en la creación de la escuela de dibujo y náutica en el año 1801, iniciándose las clases de forma provisional en el propio domicilio de Smith (Adserà, 1993, p. 50). En 1808 la escuela se abrió a la enseñanza de las niñas tarraconenses de forma gratuita por iniciativa de Smith (Rovira, 1982, p. 135-136). Su preocupación por la enseñanza hizo que se interesasen por la obra de uno de los más avanzados pedagogos de su tiempo, el suizo Johann Heinrich Pestalozzi, tal y como se refleja en una carta que recibió Martí en la que se habla del envío de una obra de este pedagogo suizo para Smith (Quintana, 1992, p. 79).

\section{LOS MÉDICOS AMIGOS DE MARTÍ Y LA DIFUSIÓN DE LA VACUNA}

El prestigioso médico Francec Salvà (Figura 3) que como hemos visto fue un gran amigo de Martí, fue un gran defensor de la variolización que se hacía en Europa desde principio de siglo XVIII, pero que había desencadenado una fuerte polémica sobre los riesgos que entrañaba. La primera variolización en Barcelona tuvo lugar en el año 1776, y un año después Salvà ya la defendía públicamente en la obra $L a$ inoculacion presentada a los sabios, y antes del final del siglo había mantenido diversas polémicas en defensa de esta técnica ante varios detractores, como el médico austríaco Anton de Haen, el presbítero Vicente Ferrer Gorraiz, o el médico Jaime Menós (Gorina, 1994, p. 16). Como hemos dicho Smith también debería conocer la existencia de esta técnica a través del médico irlandés O'Scanlan.

En el año 1796 Edward Jenner iniciaba sus experiencias de vacunación, que hizo públicas dos años más tarde en la obra An inquiry into the causes and effects
Figura 3. Retrato de Francesc Salvà i Campillo. Fundación Española para la Ciencia y la Tecnología, Eulogia Merle.

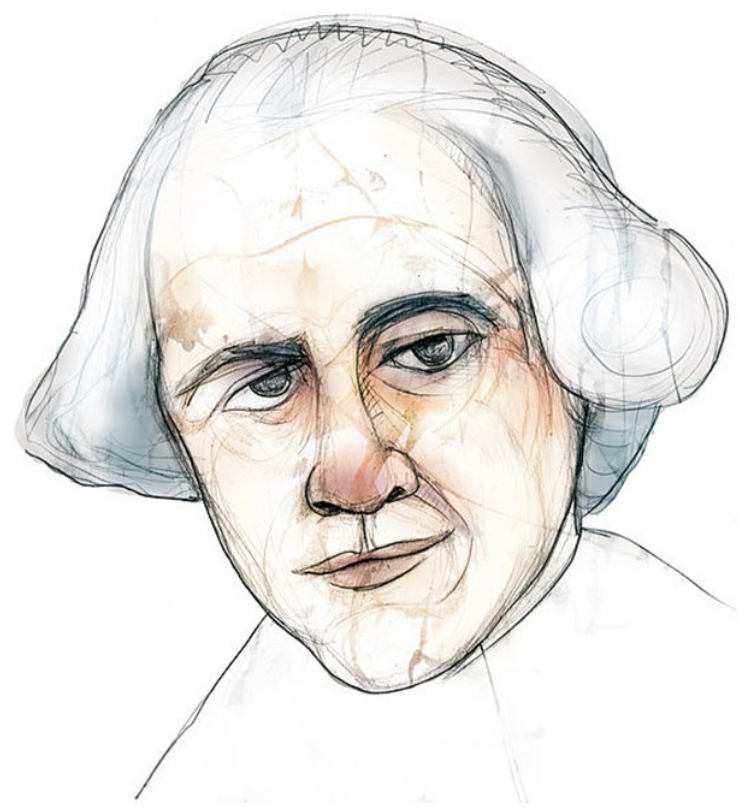

of the variola vaccine. Salvà quedó fuertemente impresionado por las mejoras introducidas por Jenner defendiendo desde entonces la práctica de la vacunación, aunque con ciertos matices. Su gran admiración por el médico inglés le llevó a dedicarle parte del discurso inaugural del curso clínico del año 1806 (Janer, 1832, p. 12 y 35). Además en 1801 impulsó y financió un premio a la mejor disertación sobre las ventajas o inconvenientes de la inoculación de la vacuna, que concedería la academia médica barcelonesa (Pérez, 2007, pp. 133, 134).

Otro amigo médico de Martí también miembro de la academia médica de Barcelona, Francesc Piguillem, tuvo un gran protagonismo ya que fue el introductor de esta nueva técnica en España realizando las primeras vacunaciones en Puigcerdà en diciembre del año 1800, usando el material que le había enviado el médico francés François Colon desde París. Piguillem en el año 1801 se esforzó en divulgar la nueva técnica a través dos obras, la traducción de una obra del doctor Colon que apareció con el título Ensayos sobre la inoculación de la vacuna, y un libro escrito por él, La vacuna en España o cartas familiares sobre esta nueva inoculación, donde se refiere a la vacunación en Tarragona (Piguillem, 1801, p. 58). A pesar de que la Academia Médico Práctica de Barcelona fuera una de las instancias impulsoras de la vacunación, en los primeros años criticó a Piguillem por su excesiva 
confianza en el joven médico francés François Colon en un informe que entre otros firmó Salvà (Olagüe y Astrain, 2004, p. 14). Esta desconfianza de Salvà hacia Piguillem también se pone de manifiesto en una carta sobre la vacuna que le envió al médico Josep Xirau en 1803, y que otro médico, Joan Puig i Mollera recogió en una obra publicada el mismo año:

Si se tratase de dar premios por haber escrito à CoIon, que enviase vacuna de París, y por haberla repartido después, bastaria poco tiempo; pero la Academia quiere premiar à los que den pruebas autenticas de haber exâminado à fondo esta practica (Puig, 1803, p. 47)

Otro miembro de la academia y también amigo de Martí, Francesc Carbonell, hizo de censor de una carta sobre la vacuna que envió el médico de París Alphonse Leroy (Corbella, 2003, p. 49). Leroy era un polémico personaje que después de haber defendido inicialmente la vacuna se convirtió en un destacado opositor que tuvo cierta repercusión en la península, tal y como puede comprobarse en la obra que publicó al año siguiente el médico y cirujano Diego de Bances, uno de los miembros del núcleo vasco-navarro impulsor de la vacuna (Bances, 1802, pp. 71-88).

En mayo de 1801 el catedrático de anatomía del colegio de cirugía de Barcelona Esteve Marturià, presentó en una junta literaria de esta institución la comunicación "Disertación sobre el uso de la vacuna", con el objeto de divulgar esta nueva técnica. En las páginas finales del manuscrito puede observarse como los dos cirujanos que hemos explicado que intercambiaron material botánico con Martí, Bas y Torner, se mostraron suspicaces en relación a este nuevo procedimiento. Bas mostró reparos al hecho de inocular sustancias procedentes de una especie distinta de la humana, y Torner se refirió a que todavía se disponía de pocos datos².

La influencia que pudieron ejercer sobre Martí los distintos médicos y cirujanos amigos suyos que trataron el tema de la vacunación así como la gran repercusión que tuvieron en toda Europa los trabajos de Jenner, pudieron ser decisivos para que el naturalista de Altafulla se interesase por esta nueva técnica en su viaje por las principales capitales europeas durante los años 1800 y 1801, como comprobaremos más adelante.

\section{LA VACUNACIÓN EN TARRAGONA}

La primera vacunación en Tarragona se produjo en mayo de 1801 gracias a los esfuerzos realizados por Juan Smith y usando el material que le proporcionó el doctor Piguillem (Smith, 1801, p. 9). La información más completa sobre esta temprana vacunación la en- contramos en un librito que lleva por título Progresos de la vacina en Tarragona (Figura 4). Está firmado con las iniciales D.J.S.B.D.L.R.A., que parecen corresponder a Don Juan Smith Brigadier de la Real Armada. Esto queda confirmado en el ejemplar que poseía Fèlix Torres Amat puesto que muestra su ex libris, y forma parte del fondo Torres Amat que se encuentra en la Biblioteca de Catalunya. En este ejemplar podemos leer la siguiente anotación manuscrita en la portada: "Juan Smith Brigadier \&", lo cual reafirma su autoría. Torres Amat fue un destacado eclesiástico, helenista e historiador de la literatura, senador por Barcelona y obispo de Astorga, y que fue acusado de filojansenismo. Además, mantuvo una gran amistad con Martí (Camós, 2013b) y residía en Tarragona en los primeros años del siglo, por tanto también debería haber conocido a Smith. Esta obra es uno de los primeros libros originales sobre la vacuna publicado en el Estado. Consta de 48 páginas y en la misma no figura la fecha de publicación. Sin embargo sabemos que apareció en el mismo año de la vacunación, 1801, puesto que Smith antes de final de este año ya la había enviado a la Real Sociedad Económica de Amigos del País de Valencia $^{3}$ y encontramos una referencia a la obra en el Diario de Madrid del 23 de noviembre de 1801 que comentaremos más adelante.

Es necesario destacar el singular perfil del escritor del libro. De los autores de los 48 textos sobre la vacunación publicados en el Estado español entre 1800 y 1805 , destacan una mayoría de médicos y cirujanos, y algunos eclesiásticos y burgueses ilustrados; al ingeniero militar Juan Smith habría que situarlo entre los cinco autores que no tenían ninguna de las ocupaciones citadas (Olagüe y Astrain, 2004, p. 21). El autor era consciente de que el hecho de no pertenecer a la profesión médica podría hacer perder autoridad a su obra, por lo que trata de justificarse argumentando por un lado la sencillez del procedimiento, y por otro que diversos autores que no pertenecían al mundo de la sanidad ya habían divulgado la vacuna en Inglaterra y en Francia:

Aunque esta pueda parecer oficiosidad fuera de lugar en quien no es facultativo la sencillez del medicamento era de naturaleza à quitar este reparo, y mas con el exemplo de varios que en Inglaterra y en Francia sin serlo se han hecho un honor de propagarlo (Smith, 1801, p. 8).

Más adelante vuelve a referirse a la cuestión afirmando que sin la participación en la difusión de aquellos que no eran sanitarios el proceso de propagación de la nueva técnica habría sido mucho más lento: 
Figura 4. Portada del libro de Juan Smith perteneciente a Fèlix Torres Amat que se encuentra en fondo Torres Amat de la Biblioteca de Catalunya, donde puede apreciarse escrito a mano el nombre de Juan Smith, Brigadier.

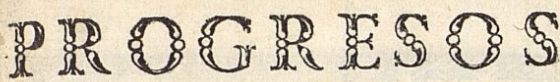

\section{DE LA V.ACINA}

\section{EN TARRAGONA}

\section{ó}

\section{INSTRUCCIONES Y REFLEXIONES} sucintas sobre la inoculacion de la Vacina dirigidas á los Padres de familia y á los sugetos que sin ser facultativos se quieran dedicar al fomento $y$ propagacion de este admirable

descubrimiento, en beneficio de la humanidad.

$$
\text { Inan fmith, Bxightion \& }
$$

Por D. F. S. B. D. L. R. A.

- CONLICENCIA:

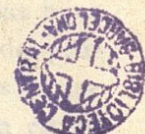

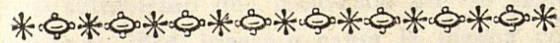

Tarragona: En la Imprenta de María Ca-

aals Viuda, administrada por Miguel Puigrubí.

\section{$C B 1001975561$}

Si en ninguna parte después del descubrimiento de Jenner se hubiese practicado la vacina sino por sujeto experimentado en ella ¿quan lentos no hubieran sido sus progresos? ¿y quantos años no se hubiera tardado en extenderla por Europa? quando en el dia se puede decir que el correo la ha llevado a todas partes (Smith, 1801, p. 33).

Smith contó con la colaboración de tres médicos que fueron los que hicieron las vacunaciones, aunque nos dice en la obra que él también estaba presente siempre que podía. El médico que colaboró más intensamente con Smith fue Manuel Dalmau Capdevila, nacido en Tàrrega y médico del hospital de Tarragona y del arzobispo Armanyà. También lo hizo su hijo Manuel Dalmau Magnífic, miembro de la Junta de Sanidad de Tarragona. El tercer facultativo fue Joan Vives, médico de una pequeña población cercana a Tarragona llamada Constantí.
El texto de Smith se inicia con una corta introducción donde describe la viruela y sus terribles efectos, el antiguo modo de prevenirla a través de la variolización y las mejoras producidas por la nueva técnica introducida por Jenner. Expone después las gestiones hechas para obtener la vacuna que culminaron con la obtención del material que le fue enviado por el doctor Piguillem desde Barcelona, quién para Smith se convirtió un referente puesto que lo cita en seis ocasiones. La parte central de la obra y la más extensa la dedica a explicar de forma detallada la manera corecta de vacunar. En las últimas páginas insiste en las enormes ventajas que tiene la vacuna de Jenner frente a la variolización. Por el contenido de la obra parece que la intención de Smith era divulgar de una forma llana la importancia del nuevo método de prevención y la manera práctica de hacer las vacunaciones.

A través de esta obra sabemos que la vacunación se inició el 3 de mayo de 1801, motivada por la existencia de un foco de epidemia de viruela en una población situada a tres cuartos de legua de Tarragona (Smith, 1801, p. 9). Esta primera vacunación la hizo el médico de Constantí Joan Vives, por lo que suponemos que fue en esta población vecina de Tarragona donde se produjo el primer foco de la epidemia. Clave para el éxito de la vacunación hubo de ser que los primeros inoculados fuesen los cuatro hijos del gobernador Mariano lbáñez, y que ninguno de los cuatro sufriera graves consecuencias posteriores, a pesar de que la hija pequeña en los días siguientes tuviera fiebre a causa de la salida de los dientes. Las vacunaciones que se realizaron posteriormente las hizo el doctor Manuel Dalmau Capdevila, inoculando gratuitamente en su casa a cualquiera que lo pidiera, y contando con la ayuda de su hijo que también era médico.

Según Smith, Manuel Dalmau Capdevila fue fundamental para la buena acogida de la vacuna por parte de la población. Fue él quien hizo el dictamen de la causa de la fiebre que sufrió la hija pequeña del gobernador días después de su vacunación cuando algunos ya la atribuían al contagio de la viruela, lo que hubiera puesto en grave peligro la aceptación de la vacuna por parte de otros ciudadanos. Además fue un ardoroso divulgador intentando persuadir a la población de la necesidad de vacunarse de formas tan sorprendentes como pregonándolo desde los balcones:

... se fatigaba en persuadir à todos, gritando como lo he visto desde los balcones, y exponiendo à los padres y madres el riego que amenazaba a sus hijos con la epidemia de viruelas naturales que ya se había introducido en la Ciudad (...) Es preciso confesar que, a no haber 
sido por su dictamen y persuasiva que era de mucho peso, no se hubiera podido propagar este remedio en Tarragona (Smith, 1801, pp. 12,13).

Tal como se explica en la obra, uno de los grandes problemas era distinguir las verdaderas vacunas de las falsificaciones, que parece que abundaron por toda Europa, llegando a producir situaciones muy graves como en el caso de las falsas vacunas usadas en Ginebra que causaron muchos muertos y que Smith relata (1801, p. 31). Y es en relación con esta problemática donde aparece Martí como un experto que podía garantizar la calidad de la vacuna, sabiendo distinguir los granos que se producían a consecuencia de la inoculación de la verdadera vacuna de los que se producían por la variolización o por falsas vacunas:

... tenemos el consuelo de que Don Antonio Martí vecino de esta ciudad que acaba de ver los vacinados de los hospitales de Londres y París nos ha confirmado en la seguridad de que la verdadera vacina es la que tenemos aquí, y que los granos de estos inoculados son idénticos a los que vió en dichos hospitales (Smith, 1801, pp. 34).

Sabemos que Martí viajó por las principales ciudades europeas durante 1800 y 1801, donde visitó universidades, academias e institutos. Según Parcet (1907, p. 696) mantuvo contactos con «los sabios más notables de aquella época», y según Torres Amat (1836, p. XXII) «a principios de este siglo recibió en Londres y París singulares muestras de aprecio de parte de los sabios de aquellas academias». Sabemos muy poco de este viaje, pero a partir de lo referido por Smith podemos afirmar que durante el mismo visitó hospitales en Londres y París interesándose por la nueva técnica de la vacunación.

En el texto de Smith destaca la claridad con que expone la forma de distinguir el resultado de la vacunación hecha con la vacuna auténtica de la realizada con la vacuna fraudulenta. De ello se hizo eco el cirujano Juan de Azaola en un artículo publicado el 23 de noviembre de 1801 en el Diario de Madrid. El artículo se publicó a raíz de una fuerte polémica que se produjo en Madrid a consecuencia de que el niño Mateo Luquet al poco de ser vacunado sufrió la viruela (Duro, 2014, pp. 244-247). Esto provocó una campaña en contra de la efectividad de la vacunación y de su responsable, el cirujano Juan de Azaola. Éste se defendió argumentando que el niño había enfermado porque le había puesto una vacuna falsa tal como ya había supuesto desde el principio, y así lo había comunicado a sus padres. Para demostrar que era capaz de diferenciar las vacunas legítimas de las falsas se refirió a diferentes obras, entre ellas la de Smith:
En el folleto titulado Progresos de la Vacuna en Tarragona, impreso en la misma ciudad por el Sr. D. Juan de Smith, Brigadier de la Real Armada, que ha vacunado 1400 personas con toda felicidad, y que ha escrito un tratadito que le hace mucho honor, dice en la pag. 28. Así como hay viruelas locas ó espurias hay vacuna falsa ó bastarda, y del mismo modo que aquellas, no preserva ésta de viruelas naturales ${ }^{4}$.

Esta referencia a la vacunación en Tarragona incluye una notable confusión al dar a entender que las vacunaciones las había hecho el propio Smith, cuando como hemos visto las hicieron tres médicos, Dalmau padre e hijo y Vives. Cabe resaltar que Azaola también se refiere a la gran cantidad de vacunados en Tarragona cifrándolos en 1400 personas. En los Progresos de la Vacuna en Tarragona, Smith se refiriere a que Martí también se había sorprendido de la gran cantidad de vacunados en la ciudad que no había visto en la misma proporción en ninguna población francesa, por lo que conocemos que Martí no solo se interesó por la vacunación en los hospitales de Londres y París, sino también por su difusión en Francia:

También nos ha manifestado que proporcionalmente à la población en ninguna parte de Francia se halla la Vacina tan propagada como lo está en Tarragona (Smith, 1801, p. 34).

El proceso tuvo que ser muy exitoso, puesto que si hacemos caso a la carta que el 12 de setiembre de 1801 la Condesa de Montijo envió a Ruiz de Luzuriaga, la cifra de vacunados en aquella fecha ya era de 1900 (Riera y Rigau, 1992, p. 297). Se trata de una cifra extraordinaria si tenemos en cuenta que la población de Tarragona y alrededores no debería ser de mucho más de 15.000 habitantes. Si consideremos que según Ruiz de Luzuriaga hasta finales de 1801 se habían vacunado 861 personas entre Madrid y Aranjuez (Olagüe y Astrain, 2004, p. 24), podremos ver la gran importancia por lo menos cuantitativa de este foco de vacunación en la península. A Madrid llegaron pocas noticias de este destacado núcleo de vacunación, probablemente a causa de la falta de buenas medidas administrativas y de coordinación.

Algunos autores han afirmado que Martí informó a la Academia Médico Práctica de Barcelona sobre los progresos de la vacunación en Londres y París, y que se mostró abiertamente partidario de la nueva técnica (Olagüe y Astrain, 2004, p. 15). Esto se apoyaría en el texto de la obra de Smith y en lo que afirma Quintana sobre la relación de Martí con la citada academia (1935, p. 155). Es lógico suponer que esto se hubiera podido producir a pesar del miedo de Martí 
a hacer públicas sus ideas. Sin embargo, más allá de los datos expuestos anteriormente no se ha localizado en los textos mencionados ninguna referencia a algún escrito en el cual Martí apoye diáfanamente la vacunación, ni un informe suyo sobre la vacunación en Londres y París. Por tanto no podemos confirmar estas afirmaciones.

Otro médico y cirujano amigo de Martí vinculado con la vacunación fue Jaume Parcet. En la necrología que escribió sobre Martí explica que habían sido muy amigos durante los últimos 26 años de su vida, que tenía entrada libre a su gabinete reservado en Tarragona, y que con él había mantenido largas conversaciones relativas a las ciencias naturales (Parcet, 1907, p. 700). Él también fue un defensor de la vacunación puesto que inoculaba en su casa tal como se puede comprobar en diversos anuncios que aparecieron en el apartado de "Avisos" del Diario de Tarragona a lo largo del año 1809. Así, el de 5 de mayo de aquel año apareció el siguiente aviso: «Hoy se Vaccinará. El que quiera aprovecharse de este seguro precautivo de viruelas acuda a las tres de la tarde a casa de Jaime Parcet Cirujano del Hospital de esta Ciudad ${ }^{5}$. Aunque comenzó a ejercer en el Hospital de Sant Pau i Santa Tecla de Tarragona en 1804, por tanto tres años después de este primer episodio de vacunación al que nos hemos referido, el gran respeto que le tenía a Martí que se pone de manifiesto en su necrología, nos permite pensar que el naturalista de Altafulla pudo influir en el decidido posicionamiento de Parcet en favor de la vacunación.

Smith hizo verdaderos esfuerzos para divulgar el nuevo método de la vacuna. Al final de Los progresos de la vacina en Tarragona expone las dificultades que existían para que las noticias de este nuevo descubrimiento llegaran a los pueblos del interior de la península así como a los que no eran muy importantes. Para solucionarlo se mostraba dispuesto a efectuar él mismo la difusión haciendo llegar su obra de forma gratuita a todas las provincias y partidos:

... persuadido de que los conocimientos y descubrimientos caminan lentamente con particularidad à los pueblos interiores y de segundo orden, me ha parecido conveniente escribir estas instrucciones y noticias, aunque succintas, suficientes; para que llegue à noticias de todos este admirable descubrimiento. Al mismo intento he determinado remitir gratuitamente à todas las provincias y partidos exemplares de ellas. De esta manera sabrán à lo ménos todos que existe este remedio, y persuadidos de su eficacia no dexarán de poner los medios para promover su uso (Smith, 1801 , pp. 47 y 48 ).
A pesar de los esfuerzos que Smith se muestra dispuesto a realizar, la vacunación en Tarragona tuvo un eco bastante limitado. Parece que sí lo tuvo en Valencia cuando en mayo de 1801 envió a la Real Sociedad Económica de Amigos del País de Valencia una carta explicando cómo se vacuna y adjuntando el material para realizarlo, y haciendo llegar su obra antes de finalizar el año. A consecuencia de esto la sociedad dio a conocer la posibilidad de vacunar a quien lo solicitase, y en la junta de diciembre de 1801 se explica que en la capital y en los pueblos cercanos «hay un crecidísimo número de vacinados ${ }^{6}$. Además sabemos que también llegaron las instrucciones de Smith y la vacuna a la Sociedad Aragonesa de Amigos del País (Olagüe y Astrain, 1995, p. 16), y que las noticias llegaron a Cartagena (Smith, 1801, p. 15) y a Madrid. Hasta el momento no hemos encontrado más datos que puedan corroborar hasta qué punto se cumplió la voluntad de Smith de hacer llegar su obra a todas las provincias. Sin embargo sabemos que el trabajo de Smith era conocido en Vizcaya por un personaje que guarda bastante paralelismo con el brigadier. Se trata de Lope García Mazarredo, quien sin ser sanitario también publicó en 1801 un pequeño opúsculo titulado Instrucciones prácticas para la inoculación de la vaccina, que era la traducción de un escrito de médico francés Henri Marie Husson. García Mazarredo debía conocer las vacunaciones que se estaban realizando en Tarragona y el protagonismo de Smith, puesto que en octubre de 1801 envió al ayuntamiento de esta ciudad dos ejemplares del opúsculo con instrucciones precisas para que uno de ellos llegara a manos del brigadier (Riera, 1992, p. 300).

Más sorprendente es la llegada de la obra a Puerto Rico. Muy probablemente la posibilidad de comerciar con América desde el puerto de Tarragona establecida en el año 1800, facilitó que la obra de Smith pudiera llegar a las colonias españolas de aquel continente. Tenemos constancia de que el librito ya estaba en Puerto Rico en el año 1804, cuando la Real Expedición Filantrópica de la Vacuna llegó a esta isla y se encontró con que el doctor Oller ya había iniciado la vacunación con material proveniente de la isla Saint Thomas, en aquel momento colonia danesa. El director de la Real Expedición, el cirujano Francisco Balmis, contrariado porque las vacunas usadas no provinieran de colonias españolas y no fiándose de la calidad de las mismas, acusó a Oller de fraude e incompetencia; y éste se defendió detallando las obras de referencia que había usado para informarse, y entre ellas cita a «la dada á luz en Tarragona pr. un incognito» (Riera y Rigau, 1992, p. 298). La obra no podía ser otra que el libro Progresos de la vacina en Tarragona, y el "incógnito" Juan Smith Sinnot. 


\section{CONCLUSIONES}

Juan Smith Sinnot, brigadier de la Armada, prestigioso ingeniero y director de las obras del puerto de Tarragona, fue el máximo responsable de la temprana vacunación de más de un millar de tarraconenses que se produjo en la primavera del año 1801. Para ello hubo de ser determinante su traumática experiencia de sufrir en soledad la viruela durante su juventud, su buena formación científica, su curiosidad por conocer los avances en diversos campos de la ciencia, así como su gran sensibilidad social. No solo hizo las gestiones necesarias para recibir el material del introductor de la vacuna en el Estado español, Francesc Piguillem, sino que hizo notables esfuerzos para difundir el nuevo método preventivo a través de la publicación de una pequeña obra, y enviando noticias, material y el libro a diferentes puntos de la península.

\section{NOTAS}

1. Carta de D. Juan Smith entregando a la Sociedad varias obras que a esta finalidad le habían sido remitidas por D. Gabriel Ciscar, Archivo de la Real Sociedad Económica de Amigos del País de Valencia, Caja 27 - Legajo II - Signatura 3, https://riunet.upv.es/bitstream/handle/10251/19345/27_ II_3.PDF?sequence $=1$ \&isAllowed $=y$

2. Marturià, Esteban (1801), Disertación sobre el uso de la vacuna, Juntas literarias del colegio de cirugía de BarceIona, BUB-reserva, Caja 7. Doc. 86, http://mdc.cbuc.cat/ $\mathrm{cdm} /$ singleitem/collection/juntasliter/id/34/rec/7

\section{BIBLIOGRAFÍA}

Adserà, Josep (1993), Joan Smith i Sinnot: director del port modern de Tarragona: 1800-1809, Tarragona, Autoritat Portuària de Tarragona.

Amenedo, Mónica (2010), “Timoteo O’Scanlan: Vida pública y privada en El Ferrol ilustrado", Garoza, 10, pp. 9-22, [en línea], disponible en: http://webs.ono.com/garoza/G10Amenedo.pdf [consultado el 23/01/2017]

Bances, Diego de (1802), Tratado de la vaccina, o viruela vacuna transmitida al genero humano, Pamplona, Imprenta de la viuda de Longás é Hijo.

Camós, Agustí (2013a), "Antoni de Martí i Franquès, la generación espontánea y la transformación de los organismos", Asclepio, 65 (2), pp. 1-13, [en línea], https://doi.org/10.3989/ asclepio.2013.22

Camós, Agustí (2013b), “Antoni de Martí i Franquès i Fèlix Torres Amat: Ciència i dissidència religiosa a la Catalunya d'inici del segle XIX", Actes d'història de la ciència i de la Tècnica, 6 (1), pp. 1-32, [en línea], https://doi.org/10.2436/20.2006.01.178
Otro destacado ciudadano de Tarragona, el científico Antoni de Martí, también estuvo relacionado con esta vacunación. Además de preocuparse por temas médicos como la relación que podía existir entre el aire atmosférico y las enfermedades y por la fiebre amarilla, también se interesó por la vacuna. Este interés pudo deberse en parte a la defensa de la vacunación que hicieron algunos de sus amigos médicos, como Francesc Salvà y Francesc Piguillem. A través de la obra de Smith Progresos de la vacina en Tarragona, conocemos que Martí visitó hospitales en Londres y París donde se hacían vacunaciones, y que era capaz de diferenciar a través de las pústulas que producían las buenas vacunas de las fraudulentas. También fue consciente de la importancia cuantitativa de la vacunación que se estaba haciendo en Tarragona en el año 1801 , si se comparaba con las realizadas en Francia en la misma época.

3. Real Sociedad Económica de Amigos del País de Valencia (1802), Junta publica de la Real Sociedad Económica de amigos del país de Valencia celebrada el dia 9 de diciembre de 1801, Valencia, Imprenta de D. Benito Monfort, pp. 33-34.

4. Azaola, Juan de, "Señor Diarista", Diario de Madrid, 23 de novembre de 1801, n. 327, p. 1339.

5. “Avisos”, Diario de Tarragona, 9 de mayo de 1809, n. 130, p. 532.

6. Obra citada en la nota 2, p. 34 .

Camós, Agustí (2016a), “Antoni de Martí i Franquès cun genio aislado? La llegada del lamarckismo a Barcelona en la primera mitad del siglo XIX", Dynamis, 36(2), pp. 391-417, disponible en: http://www.raco.cat/index.php/Dynamis/article/ view/313562/403680 [consultado el 14/3/2018]

Camós, Agustí (2016b), “Antoni de Martí i Franquès i el debat sobre la febre groga que tingué lloc a Barcelona en els primers anys del segle XIX", Actes d'història de la ciència $i$ de la Tècnica, 9, pp. 105-136, [en línea], https://doi. org/10.2436/20.2006.01.197

Chaptal, Jean-Antoine (1816), Química aplicada a las Artes, traducido por Francesc Carbonell, Barcelona, Imprenta de Brusi.

Corbella, Jacint (2003), "Sobre alguns aspectes médics de l'obra de Francesc Carbonell i Bravo (1768-1837): La seva tasca a I‘Academia de Medicina”, Gimbernat, 39, pp. 29-53.

Delgado, José A. (1985), Catálogo de pruebas de nobleza del Real Colegio de San Telmo de Sevilla, Madrid, Ediciones Hidalguía. 
Duro, José L. (2014), Los inicios de la lucha contra la viruela en España. Técnica e ideología durante la transición de la inoculación a la vacuna (1750-1808), tesis doctoral dirigida por José V. Tuells, Universitat d’Alacant, [en línea], disponible en: http://rua.ua.es/ dspace/handle/10045/45825 [consultado el 23/01/2017]

Escoda, Coia (2008), “Los ingenieros militares partícipes de las obras del Puerto de Tarragona en el siglo XVIII", Revista de Historia Naval, 102, pp. 61-73.

Gorina, Núria (1994), "La profilaxi de la verola. La seva difusió a Catalunya durant el set-cents", Enrahonar, 22, pp. 9-19, [en línea], disponible en: https://ddd.uab.cat/pub/enrahonar/02 11402Xn22/0211402Xn22p9.pdf [consultado el 23/01/2017].

Grau, Josep (2011), "La química de l'aire". En: Martí, Antoni, La química de l'aire, Josep Grau y Josep Bonet (eds.), Tarragona, Publicacions URV, pp. 15-46.

Hernández, Bonaventura y Morera, Francisco (1865), Descripción histórica de las estatuas, medallones, bajos relieves y bustos que adornan el frontispicio del palacio de las Exmas. Corporaciones Diputación Provincial y Ayuntamiento de la fidelísima y ejemplar ciudad de Tarragona, Tarragona, Imprenta de los Sres. Puigrubí y Arís.

Janer, Fèlix (1832), Elogio histórico del Dr. D. Francisco Salvá, Barcelona, Imprenta de Joaquín Verdaguer.

Maier, Jorge (2003), Noticias de antigüedades de las actas de sesiones de la Real Academia de la Historia (1792-1833), Madrid, Real Academia de la Historia.

Martí, Antoni (2011), La química de l'aire, Josep Grau y Josep Bonet (eds.), Tarragona, Publicacions URV.

Olagüe, Guillermo y Astrain, Mikel (1994), "Una carta inédita de Ignacio María Ruiz de Luzuriaga (1'763-1822) sobre la difusión de la vacuna en España (1801)", Dynamis, 14, pp. 305-337, [en línea], disponible en: http://www.raco.cat/index.php/Dynamis/article/view/105961/149966 [consultado el 23/01/2017]

Olagüe, Guillermo y Astrain, Mikel (1995), “Propaganda y Filantropismo: los primeros textos sobre la vacunación jenneriana en España", Medicina e Historia, 56, pp. 5-28.

Olagüe, Guillermo y Astrain, Mikel (2004), "iSalvad a los niños!: Los primeros pasos de la vacuna antivariólica en España (1799-1805)", Asclepio, 56 (1), pp. 7-31, [en línea], https:// doi.org/10.3989/asclepio.2004.v56.i1.70

Parcet, Jaume (1907), "Necrología de Dn. Antonio Martí y Franqués escrita por el doctor Jaime Parcet, leída el 20 noviembre 1832", Boletín Arqueológico de Tarragona, 20 (1), pp. 695-700.

Pereyra, Pedro (1800), Estado general de la Real Armada: año de 1800, Madrid, Imprenta Real.

Pérez, Núria (2007), Anatomia, química i física experimental al Reial Col-legi de Cirurgia de Barcelona (1760-1808), te- sis doctoral dirigida por Àlvar Martínez, UAB. www.tdx. cat/bitstream/10803/5174/1/npp1de1.pdf [consultado el 23/01/2017].

Piguillem, Francesc (1801), La vacuna en España o cartas familiares sobre esta inoculación, Barcelona, Sierra y Olivér Martí.

Puig, Juan (1803), Aviso importante sobre los casos extraordinarios de viruela legítima sobrevenidos mucho tiempo despues de la vaccina verdadera, Girona, Fermin Nicolau.

Quintana, Antoni (1935), “Antoni Martí Franquès. Memòries originals. Estudi biogràfic i documental". Memòries de I'Acadèmia de Ciències i Arts de Barcelona, 3a època, 24, Barcelona, Nebots de López Robert.

Quintana, Antoni (1985), “Biografia desapassionada d'Antoni de Martí i Franquès". En: Miscel-lanea Antoni de Martí i Franquès, Tarragona, Ajuntament de Tarragona, pp. 47-88.

Quintana, Antoni (1992), “Epistolari d'Antoni de Martí Franquès", Estudis Altafullencs, 16, pp. 51-121.

Riera, Manuel (1992), "Don Juan Smith i la sopa de Rumford", Gimbernat, 17, pp. 277-288, [en línea], disponible en: http:// www.raco.cat/index.php/Gimbernat/article/view/44540 [consultado el 23/01/2017].

Riera, Manuel y Rigau-Pérez, José (1992), “Los primitivos de la vacuna en Tarragona y el ingeniero de marina Don Juan Smith", Gimbernat, 17, pp. 289-303, [en línea], disponible en: http:// www.raco.cat/index.php/Gimbernat/article/view/44541 [consultado el 23/01/2017].

Rovira, Salvador (1982), Antoni Martí i Franquès i l'Altafulla del seu temps (1750-1832), Tarragona, Centre d'Estudis d'Altafulla.

Rovira, Salvador (2011), Tarragona a la Guerra del Francès (1808-1813), Tarragona, Quaderns de l'arxiu 6.

Salvà, Francesc (1876), "Memoria segunda sobre la electricidad aplicada a la telegrafia". En: Memorias de la Real Academia de Ciencias Naturales y Artes de Barcelona no. 1ํㅗ y 2으, Barcelona, Imp. de Jaime Jepús Roviralta, pp. 41-55.

Sánchez Real, José (1998), "Tres escritos de Antonio Martí Franqués". En: Carnicer, Joan et alt, Antoni de Martí i Franquès. Recull d'articles, Altafulla, Centre d'Estudis d'Altafulla, pp. 53-59.

Smith, Juan (1801), Progresos de la vacina en Tarragona, Tarragona, Imprenta de María Canals.

Torres Amat, Fèlix (1836), Memorias para ayudar a formar un diccionario crítico de los escritores catalanes, Barcelona, Imprenta A. Verdaguer.

Tuells, José (2015), “El ensayo inédito sobre la vacuna de Ignacio María Ruiz de Luzuriaga (1763-1822)”, Dynamis, 35 (2), pp. 459480. https://doi.org/10.4321/S0211-95362015000200009 